\title{
Tandem Mass Spectrometry Study of $C$-Phenyl-N-tert-Butyl Nitrone Spin Adducts from In Vitro Rat Liver Microsomal Metabolism of Bromotrichloromethane and Carbon Tetrachloride
}

\author{
Edward G. Janzen, Hong Sang, Yashige Kotake, Coit M. DuBose, and \\ J. Lee Poyer \\ National Biomedical Center for Spin Trapping and Free Radicals, Free Radical Biology and Aging Research \\ Program, Oklahoma Medical Research Foundation, N.E. 13th Street, Oklahoma City, Oklahoma, USA \\ Masana Arimura \\ Department of Applied Chemistry, Faculty of Engineering Science, Osaka University, Toyonaka, Osaka, Japan
}

\begin{abstract}
Electron ionization and thermospray were used in conjunction with tandem mass spectrometry methods to identify trichloromethyl/C-phenyl- $N$-tert-butyl nitrone (PBN) spin adducts produced in rat liver microsomal dispersions that had been treated with reduced nicotinamide adenine dinucleotide phosphate (NADPH)-generating system and $\mathrm{BrCCl}_{3}$ (or $\mathrm{CCl}_{4}$ ). In the identification of PBN spin adducts, a scan of precursors of $m / z 57$ was utilized to confirm the presence of PBN spin adducts, because PBN spin adducts produce $\mathrm{m} / z 57$ from tert-butyl as a characteristic fragment. Use of deuterated PBN (PBN- $d_{9}$ deuterated at tertbutyl; PBN- $d_{14}$ deuterated at both phenyl and tert-butyl) improved the recognition of PBN adducts in mixtures by precursor ion scans, because $m / z 66$ (which corresponds to the deuterated tert-butyl group) is characteristic and, unlike $m / z 57$, it is not a common fragment for any other compounds. Two new PBN spin adducts that were not detected before by electron paramagnetic resonance or mass spectrometry were identified by these methods for the first time. ( $($ Am Soc Mass Spectrom 1995, 6, 847-853)
\end{abstract}

S pin-trapping has proved to be very useful for detecting short-lived radicals in chemical $[1,2]$ and biological systems [3-6]. With the spin-trapping electron paramagnetic resonance technique (STEPR), determination of the spin adduct structures has been based mainly on the hyperfine splitting (hfs) pattern of the electron paramagnetic resonance (EPR) spectra. In many cases, however, the structures of these spin adducts are difficult to determine based on their EPR spectra alone, because the hfs is often insensitive to the molecular structure of the spin-trapped radicals; for example, C-phenyl- $N$-tert-butyl nitrone (PBN)-trapped carbon centered radicals all show sixline patterns with only small differences in hfs [7]. Therefore EPR spectroscopy often is not sufficiently definitive to identify the structure of some free radicals, especially when unknown spin adducts are produced [8].

A combination of the mass spectrometric technique

Address reprint requests to Dr. Edward G. Janzen, 825 N.E. 13th, Oklahoma City, Oklahoma 73104. with ST-EPR is being developed to characterize the structures of free radicals involved in a variety of chemical and biochemical reactions [9-18]. Direct probe electron ionization (EI) mass spectrometry has been used to confirm the structures of the carbon radical adducts of $C$-(4-pyridyl-1-oxide)- $N$-tert-butyl nitrone (4-POBN) [14] formed in the metabolism of alkyl hydrazines in rat liver microsomal dispersions. The same method was used to detect the phenyl adduct of 5,5-dimethyl-1-pyroline- $N$-oxide (DMPO) in the oxidation of phenylhydrazine and in the phenylhydrazineinduced hemolysis of red blood cells $[16,18]$. The structure of the hydrazyl PBN adduct formed during microsomal metabolism of hydrazine [10] also was investigated by using probe mass spectrometry.

Analysis by gas chromatography mass spectrometry (GC-MS) of derivatized spin adducts was reported by Abe and co-workers $[13,15]$. They demonstrated that unstable spin adducts of PBN and 4-POBN may be derivatized by silylation to produce more thermally stable and volatile compounds for GC-MS. Mikami et al. [12] reported GC-MS for spin adducts of PBN and nitrosodurene formed from trapping radicals dur- 
ing the ultraviolet (UV) irradiation of the pesticide fenvalerate. Janzen et al. [11] demonstrated the use of GC-MS for the analysis of a series of alkyl spin adducts of $\mathrm{PBN}$ produced by Grignard addition to the nitrone followed by oxidation of the resulting hydroxylamine. They also studied the reaction of an aminoxyl and the corresponding hydroxylamine with silylation agents by GC-MS [9]. To improve the recognition of PBN adducts in mixtures, deuterated PBN has been used in the GC-MS analysis of spin-trapping products from the in vitro rat liver microsomal metabolism of halothane [19] and both in vivo and in vitro metabolism of carbon tetrachloride $[20,21]$.

Some radical adducts, however, are stable only in solution. High-performance liquid chromatography (HPLC) mass spectrometry offers the advantage of keeping the radicals in solution at least until they enter the ion source. Tomer and co-workers [22-26] developed a technique for the identification of radical adducts in which HPLC/EPR and liquid chromatography thermospray mass spectrometry (LC/TS-MS) or liquid chromatography electrospray ionization mass spectrometry (LC/ESI-MS) were performed under identical HPLC conditions. In their HPLC/EPR system, in addition to an UV detector, the EPR spectrometer was used to determine which peaks in the HPLC elution profile were EPR active [27-30]. Because both HPLC/EPR/ESI-MS [22, 23, 25] and HPLC/EPR/TSMS [22] utilize mild ionization techniques, ions of these intact radical species can be observed. By combining HPLC/EPR with TS, the DMPO adducts of hydroxymethyl, 1-hydroxyethyl, and hydroxyl radicals were observed [22]. By coupling HPLC/EPR with ESI, the spin-trapped radical adducts, which include phenyl, 2-, 3-, and 4-chlorophenyl and 2-bromophenyl radicals trapped with 4-POBN, and phenyl radicals trapped with 2-methyl-2-nitrosopropane (MNP), PBN, and DMPO were determined [25]. Subsequently, Tomer and coworkers [23] studied the enzymatically generated radicals of linoleic, linolenic, and arachidonic acid, trapped with nitrosobenzene and MNP. Pentyl and pentenyl radicals trapped with 4-POBN were detected by HPLC/EPR-MS in the reaction mixture of soybean lipoxygenase with linoleic, linolenic and arachidonic acid [26]. In addition, they detected ethyl and pentyl radicals by HPLC/EPR by using $4-P O B N$ in rat liver microsomal dispersions treated with adenosine $5^{\prime}$-diphosphate (ADP), reduced nicotinamide adenine dinucleotide phosphate (NADPH), and ferric chloride [31]. On the basis of LC-MS data in conjunction with the HPLC /EPR data, they determined that the structure of the carbon-centered radical from linoleic acid was 12,13-epoxylinoleic acid radical [23]. Janzen et al. [32] also have reported the mass spectra of radical adducts that use LC/TS-MS. In this study mixed double spin adducts were detected for the first time.

Trichloromethyl free radicals $\left(\cdot \mathrm{CCl}_{3}\right)$ have been detected by the spin-trapping method in rat liver when the animal is subjected to $\mathrm{CCl}_{4}$ or when rat liver microsomes are treated with NADPH and $\mathrm{CCl}_{4}$ [33-42]. With the use of HPLC and/or GC-MS, Janzen and co-workers $[20,21]$ studied the PBN-trichloromethyl adducts from rat liver extracts treated with $\mathrm{CCl}_{4}$ in vivo and in vitro. Both thin layer and liquid chromatography were used to separate a complex mixture; in addition, deuterated PBN ( $d_{9}$-deuterated on the tert-butyl group or $d_{14}$-deuterated on both phenyl and tert-butyl groups) was used to aid in the mass spectral analysis of spin adducts.

This work has been extended by using a tandem mass spectrometry method to improve the detection of the trichloromethyl-PBN spin adduct from a hexane extract of rat liver microsomal dispersions. Compared to the earlier methods reported, the tandem mass spectrometry method offers some distinct advantages. Tandem mass spectrometry reduces the need to carry out chromatographic separation. It also is simple, fast, and especially useful for the analysis of radical spin adducts that usually are present at low concentration and are not very stable for chromatography [43].

\section{Experimental}

\section{Materials}

PBN, PBN- $d_{9}$, and PBN- $d_{14}$ were obtained from OMRF Spin Trap Source (Oklahoma City, OK). All solvents used were HPLC grade. Pure $\mathrm{PBN}-\mathrm{CCl}_{3}$ and meta-hydroxy PBN were prepared and purified by column chromatography by Dr. D. L. Haire and Dr. G. Chen in the Department of Clinical Studies, University of Guelph.

\section{Sample Preparation}

Rat liver microsomal dispersions were prepared as reported previously [35]. The reaction mixture consisted of rat liver microsomal dispersions (3-mg/mL protein) in a $0.05-\mathrm{M}$ potassium phosphate buffer $(\mathrm{pH}$ $=7.4$ ), $5-$ or $25-\mathrm{mM} P B N$ (or $10-\mathrm{mM} P B N-d_{9}$ or $10-\mathrm{mM}$ PBN- $\left.d_{14}\right), 0.10-\mathrm{M} \mathrm{BrCCl}_{3}$ or $\mathrm{CCl}_{4}$, and a NADPH generating system.

The system was incubated at $37^{\circ} \mathrm{C}$ for $1 \mathrm{~h}$. After incubation, spin adducts were extracted with $10-\mathrm{mL}$ hexane, agitated by vortex, centrifuged (bench-top), and the hexane layer was separated. The concentrated extracts were used for EI mass spectrometry analyses. For HPLC/TS-MS analyses, solvent in the hexane extracts was evaporated by $\mathrm{N}_{2}$-gas flow and the residue was redissolved in $0.5-\mathrm{mL} 65 \%$ acetonitrile $\left(\mathrm{CH}_{3} \mathrm{CN}: \mathrm{H}_{2} \mathrm{O}\right.$ vol \%). Then $10-20 \mu \mathrm{L}$ was used for injection.

\section{Mass Spectrometric Analyses}

El mass spectra were obtained with a VG-Fisons Quattro triple stage quadrupole mass spectrometer (Fisons Instruments Inc., Danvers, MA). A direct mixture anal- 
ysis was performed by using an insertion probe. The source temperature was $180^{\circ} \mathrm{C}$. The electron energy employed was $15 \mathrm{eV}$. The probe temperature was 50-60 ${ }^{\circ} \mathrm{C}$.

Discharge thermospray (TS) mass spectra also were recorded with a VG-Fisons Quattro mass spectrometer. Typical operating conditions were: source temperature $250{ }^{\circ} \mathrm{C}$, nozzle temperature $120^{\circ} \mathrm{C}$, discharge current $90 \mu \mathrm{A}$. The HPLC used for the on-line LC/TS-MS system consisted of a Waters model U6k injector and Waters 510 HPLC pumps (Waters Chromatography, Milford, MA). The column was an Alltech Econosphere $3-\mu \mathrm{m} \quad 50-\mathrm{mm} \times 4.6-\mathrm{mm}$ C18 HPLC column. $\mathrm{CH}_{3} \mathrm{CN}: \mathrm{H}_{2} \mathrm{O}(65: 35)$ was used as the mobile phase at a flow rate of $0.2 \mathrm{~mL} / \mathrm{min}$.

Argon gas was used for the collision-induced dissociation (CID). The collision energy for tandem mass spectrometry analyses was $100 \mathrm{eV}$.

\section{Electron Paramagnetic Resonance Analyses}

EPR spectra of the hexane extracts were recorded by using a Bruker (Billerica, MA) ER300E spectrometer with $100-\mathrm{kHz}$ field modulation. Round quartz sample cells with $3.5-\mathrm{mm}$ i.d. were employed.

\section{Results and Discussion}

\section{Analysis of Trichloromethyl PBN Spin Adducts by Electron Ionization Tandem Mass Spectrometry}

Trichloromethyl free radicals are formed when rat liver microsomal dispersions are incubated with $\mathrm{BrCCl}_{3}$ or $\mathrm{CCl}_{4}$ in a NADPH-generating system. In the presence of PBN spin trap, trichloromethyl radicals form the aminoxyl spin adduct:

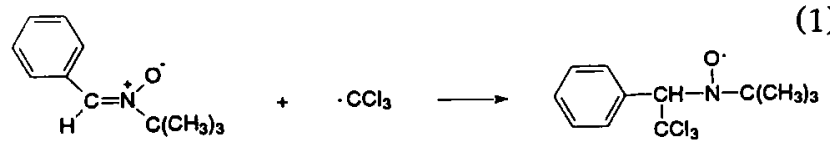

The presence of this spin adduct was confirmed by EPR in all samples in these experiments.

Assignment by mass spectrometry of the molecular ions or fragments that contain chlorine is aided by the existence of isotope peaks from ${ }^{35} \mathrm{Cl}$ and ${ }^{37} \mathrm{Cl}$ in a $3: 1$ ratio. Therefore, the isotope peaks that correspond to the $\mathrm{PBN}-\mathrm{CCl}_{3}$ spin adduct should appear at $m / z 294,296,298$, and 300 at relative intensities of 100:97.5:31.7:3.4 [44]. Figure 1 is the EI mass spectrum of a hexane extract from a rat liver microsomal dispersion incubated with $\mathrm{BrCCl}_{3}$ in the presence of $5-\mathrm{mM}$ $\mathrm{PBN}$. No molecular ions from the $\mathrm{PBN}-\mathrm{CCl}_{3}$ spin adduct are visible in the spectrum in the $\mathrm{m} / \mathrm{z} 294$ to 300 range. This is probably because the hexane extract is a complex mixture of components soluble in the organic solvent and the concentration of $\mathrm{PBN}$ spin adducts is relatively low. The weak peaks are too small to see on the scale of the strong peaks.

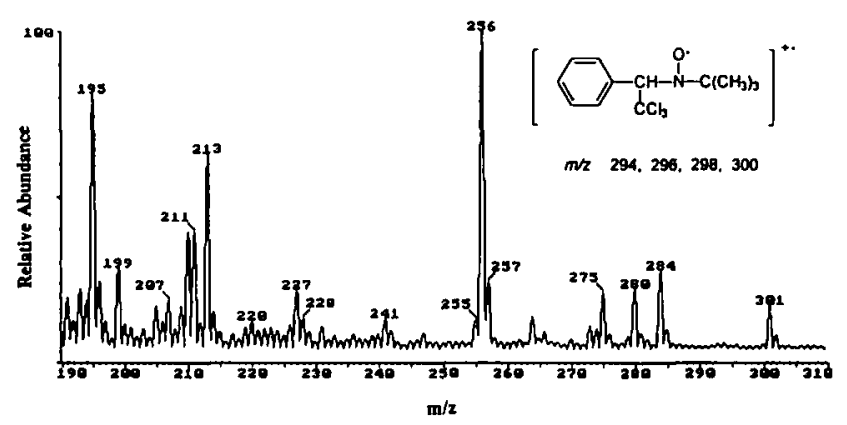

Figure 1. The EI mass spectrum of a hexane extract from rat liver microsomes incubated with $\mathrm{BrCCl}_{3}$ in the presence of 5-mM PBN.

Because PBN spin adducts usually produce an abundant fragment by EI, namely, $m / z 57\left(\mathrm{C}_{4} \mathrm{H}_{9}^{+}\right)$, a scan of precursors of $\mathrm{m} / z 57$ was recorded during tandem mass spectrometry analysis to find ions that are potentially due to PBN spin adducts. Figure $2 a$ shows a scan of precursors of $\mathrm{m} / z 57$ from the same sample as in Figure 1. Peaks that show the chlorine isotope pattern were observed at $m / z 294,296$, and 298. (The fourth isotope peak at $\mathrm{m} / z 300$ is too weak to observe in this spectrum, because its expected intensity is only $10 \%$ of the intensity of the third peak at $m / z$ 298.) The concentration of PBN in this incubation system was $5 \mathrm{mM}$; at higher PBN concentration (25 $\mathrm{mM}$ ) stronger peaks due to $\mathrm{PBN}-\mathrm{CCl}_{3}$ were detected at $m / z 294,296$, and 298, which indicated that the peak intensity was PBN-dose-dependent.

Compared to the conventional mass spectra, precursor ion spectra can exclude most of the interfering ions derived from the mixture. This approach greatly reduces the number of peaks observed in the spectrum, because only ions that produce $m / z 57$ are detected. However, there are still many other peaks observed in the spectrum because $m / z 57$ is a very common fragment for many organic compounds. For the $\mathrm{PBN}-\mathrm{CCl}_{3}$ spin adduct, the relevant precursor ions are recognized readily because of the chlorine isotope pattern. How-

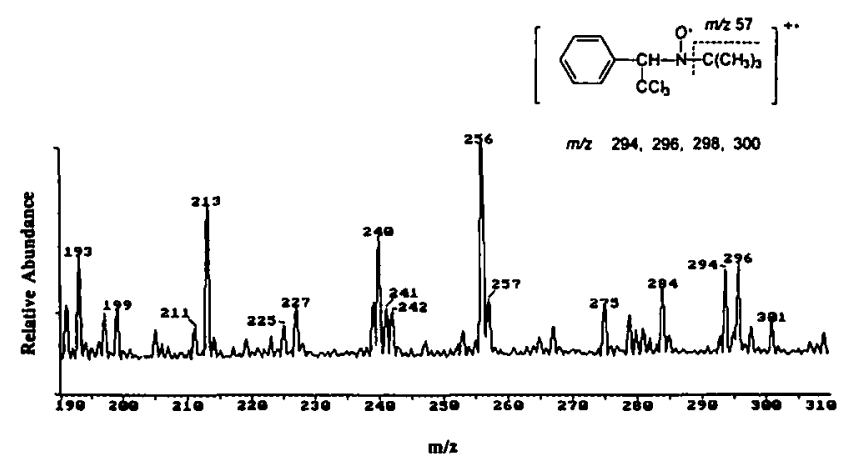

Figure 2. The scan of precursors of $m / z 57$ recorded during EI tandem mass spectrometry analysis of a hexane extract from rat liver microsomes incubated with $\mathrm{BrCCl}_{3}$ in the presence of $5-\mathrm{mM}$ PBN. 
ever, for other compounds with no abundant isotope pattern peaks or unknown spin adducts, a scan of precursors of $m / z 57$ may not distinguish which peaks in the spectrum are from PBN spin adducts.

To aid in the mass spectrometry analysis of spin adducts, deuterated PBN (PBN- $d_{9}$ ) was used. The advantage of the use of PBN- $d_{9}$ is that a major fragment ion present in the mass spectra of any $P B N$ spin adduct is $\mathrm{C}_{4} \mathrm{D}_{9}^{+}$. The fragment ion of $\mathrm{m} / \mathrm{z} 66$ (the deuterated tert-butyl group) is characteristic and, unlike $m / z 57$, it is not a common fragment for other compounds. The spectrum of precursors of $m / z 66$ from the same incubation system, but with the use of 10-mM PBN- $d_{9}$ instead of PBN, contains significantly fewer peaks (Figure 3a). The peaks at $m / z$ 303, 305, and 307 are assigned to $\mathrm{PBN}-\mathrm{CCl}_{3}$, with a shift of $9 \mathrm{u}$ compared to the nondeuterated $\mathrm{PBN}-\mathrm{CCl}_{3}(\mathrm{~m} / z$ 294, 296, and 298). As in the case of the nondeuterated PBN, the molecular ions of the trichloromethyl PBN spin adduct were not observed in the conventional EI mass spectrum. In a scan of precursors of $m / z 66$ during tandem mass spectrometry analyses of an incubation mixture when $\mathrm{CCl}_{4}$ was used instead of $\mathrm{BrCCl}_{3}$, the same result was observed except that the peaks at $m / z 249$ and 251 were much weaker (spectrum not shown).

The second set of isotope peaks observed at $\mathrm{m} / \mathrm{z}$ 288,290 , and 292 is assigned to PBN- $d_{9}-\mathrm{CCl}_{3}$ with loss of an oxygen atom and abstraction of a proton ([M $16+\mathrm{H}^{+}$). The ions of $\mathrm{m} / z 249$ and 251 are also from a PBN- $d_{9}$-spin adduct, because these two peaks shift 5 $\mathrm{u}$ when PBN- $d_{9}$ was replaced by PBN- $d_{14}$ (Figure $3 \mathrm{~b}$ ).

The peaks at $m / z 249$ and 251 in Figure 3a or $m / z$ 254 and 256 in Figure $3 b$ apparently correspond to spin adducts in which $\mathrm{PBN}-d_{9}$ or $\mathrm{PBN}-d_{14}$ has trapped free

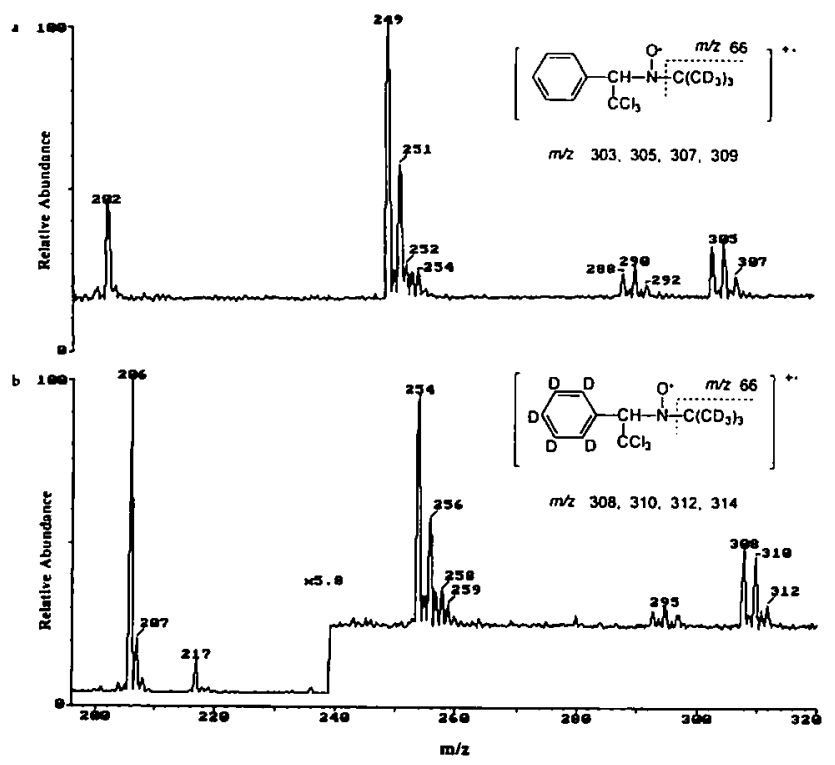

Figure 3. The scan of precursors of $m / z 66$ recorded during EI tandem mass spectrometry analysis of a hexane extract from rat liver microsomes incubated with $\mathrm{BrCCl}_{3}$ in the presence of (a) $10-\mathrm{mM}$ PBN- $d_{9}$ and (b) $10-\mathrm{mM}$ PBN- $d_{14}$. radicals of 63 and $65 u$, respectively. When nondeuterated PBN was used, the peaks that correspond to the same spin adducts also were observed at $\mathrm{m} / \mathrm{z} 240$ and 242 , with a relative intensity about 3:1 (see Figure $2 a$ ). These masses correspond to the $\mathrm{PBN}-\mathrm{COCl}$ spin adduct (Structure 1), a molecule that was postulated previously to be formed in this incubation system [42].

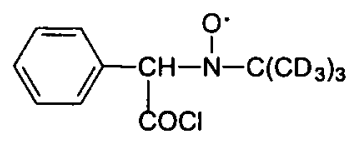

The relative intensities of these two peaks are not always the expected 100:32.5 because another peak adds to the second peak. To confirm the assignment of PBN-CCl ${ }_{3}$ and PBN-COCl spin adducts, carbon-13labeled ${ }^{13} \mathrm{CCl}_{4}$ was used instead of $\mathrm{BrCCl}_{3}$. The spectrum of precursors of $m / z 66$ (Figure 4) shows that the peaks that correspond to $\mathrm{PBN}-d_{9}-\mathrm{CCl}_{3}$ have shifted 1 $\mathrm{u}$, from $m / z$ 303, 305, and 307 to $m / z$ 304, 306, and 308 , respectively, which indicates the incorporation of the ${ }^{13} \mathrm{CCl}_{3}$ group in the adduct. The peaks at $m / z 249$ and 251 also shifted $1 \mathrm{u}$, which indicates that their corresponding spin adducts contain the carbon atom from ${ }^{13} \mathrm{CCl}_{4}$. The product ions of $\mathrm{m} / \mathrm{z} 249$ were $\mathrm{m} / \mathrm{z}$ $185\left[m / z 249-\mathrm{C}_{4} \mathrm{D}_{8}\right]^{+}, 104\left[\mathrm{C}_{6} \mathrm{H}_{5} \mathrm{CHN}\right]^{+}, 89$ $\left[\mathrm{C}_{7} \mathrm{H}_{5}^{+}\right]$, and $66\left[\mathrm{C}_{4} \mathrm{D}_{9}^{+}\right]$. The product ions of $m / z 251$ were $m / z 187\left[m / z 251-C_{4} D_{8}\right]^{+}, 89\left[C_{7} \mathrm{H}_{5}^{+}\right]$, and 66 $\left[\mathrm{C}_{4} \mathrm{D}_{9}^{+}\right]$. These observations support the assignment of the peaks to $\mathrm{PBN}-\mathrm{COCl}$.

The peak at $m / z 202$ in Figure 3a corresponds to spin adducts if $\mathrm{PBN}-d_{9}$ traps a free radical of $16 \mathrm{u}$. A peak at $m / z 193$ was observed in the same experiment when nondeuterated PBN was used. When PBN- $d_{14}$ was used instead of PBN- $d_{9}$, this peak shifted only $4 \mathrm{u}$ to $m / z 206$ (Figure 3b). Based on these mass spectra data, several spin adducts can be considered. Their structures and molecular weight are shown in Chart 1.

The molecular ions of the hydroxylamine of PBN$\mathrm{CH}_{3}$ and $\mathrm{PBN}-d_{9}-\mathrm{CH}_{3}$ would be $\mathrm{m} / z \mathrm{z} 193$ and 202, respectively; however the molecular ion of $\mathrm{PBN}-d_{14^{-}}$ $\mathrm{CH}_{3}$ would be $m / z$ 207. Therefore, the ion we detected at $m / z 202$ is not the hydroxylamine of $\mathrm{PBN}-d_{9^{-}}$ $\mathrm{CH}_{3}$. The peroxyl spin adduct [42] is expected to give benzoyl (tert-butyl) aminoxyl (PBNOX) [45]. However, the molecular ions of PBNOX and PBN- $d_{9}$ OX would be $m / z 192$ and 201, respectively, with an increase of 1 $\mathrm{u}$ for the molecular ions of the hydroxylamine of the PBNOX and PBN- $d_{9} \mathrm{OX}$; thus these two possibilities can also be excluded. The only possible structure that fits these molecular ions $(m / z 193,202$, and 206) is hydroxy-PBN, where the hydroxyl group is on the phenyl ring (ortho, meta, or para) because the mass spectrum shows that this peak is shifted by 4 not $5 \mathrm{u}$ to $m / z 206$ when PBN- $d_{14}$ is used instead of PBN- $d_{9}$ (Figure 3b).

In addition, the normal hydroxyl radical spin adduct of PBN (PBN-OH) already has been shown to be un- 


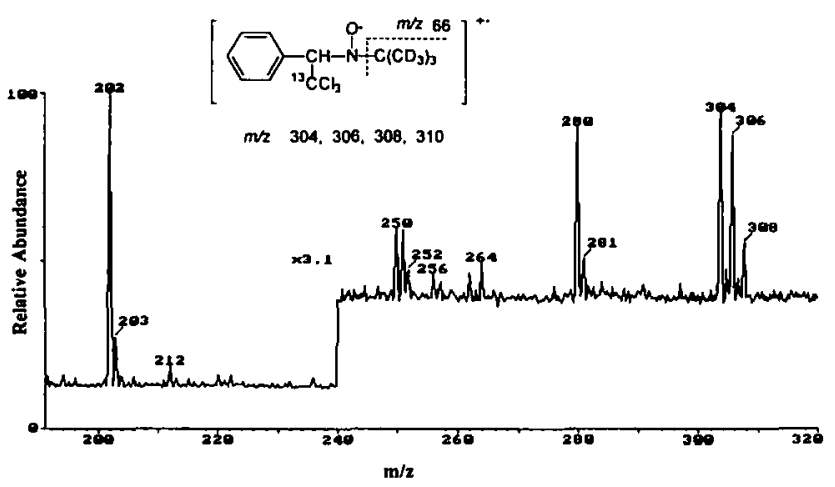

Figure 4. The scan of precursors of $m / z 66$ recorded during EI tandem mass spectrometry analysis of a hexane extract from rat liver microsomes incubated with ${ }^{13} \mathrm{CCl}_{4}$ in the presence of $10-\mathrm{mM}$ PBN- $d_{9}$.

stable; it decomposes in minutes in polar solvents to benzaldehyde and tert-butylhydroaminoxyl [46, 47]. Therefore, an "abnormal" hydroxyl radical addition to the phenyl ring is proposed so as to produce the ortho-, meta-, or para-hydroxy PBN as an eventual stable product. The generation of hydroxyl radicals in rat liver microsomal metabolism has been reported by Reinke et al [48, 49].

The product ion spectrum derived from CID of $m / z$ 202 showed peaks at $m / z 66$ and 138 (spectrum not shown); $m / z 66$ is the tert-butyl group, $\mathrm{C}_{4} \mathrm{D}_{9}^{+}$, and $\mathrm{m} / \mathrm{z} 138$ is the result of the loss of isobutylene from the precursor ion by internal deuterium transfer

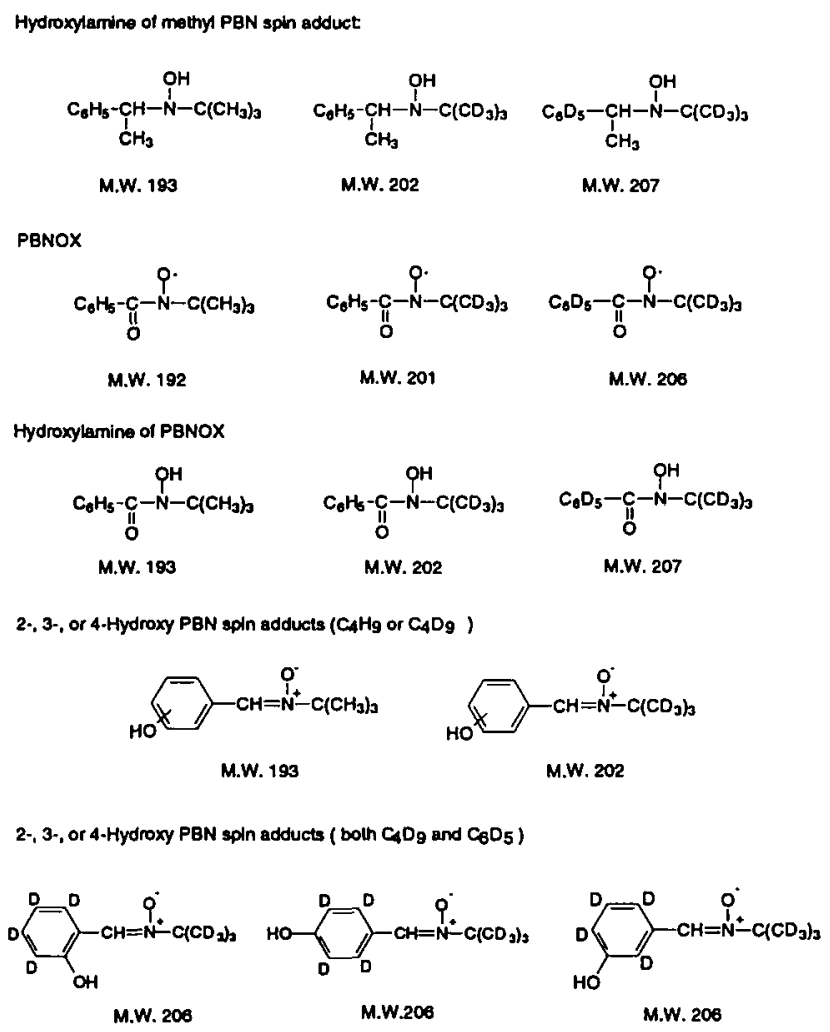

Chart 1. The possible structures of PBN spin adducts.
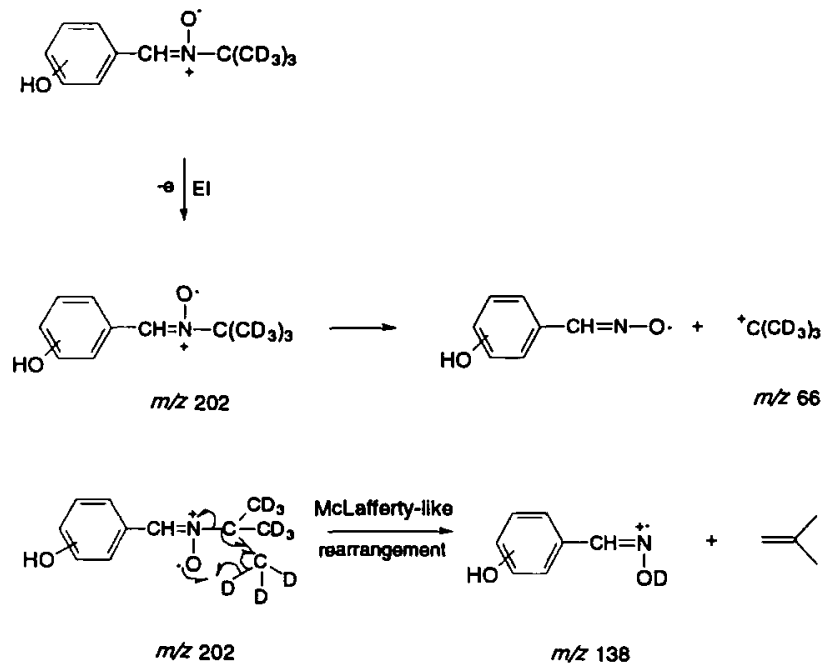

Scheme I

(McLafferty-like rearrangement [50]; see Scheme I). This assignment of hydroxy PBN was confirmed by tandem mass spectrometry of meta-hydroxy PBN.

\section{Liquid Chromatography Thermospray Mass Spectrometry}

Because Tomer and co-workers [22-26] have used LC methods to study various PBN spin adducts, LC/TSMS also was used to study the $\mathrm{PBN}-\mathrm{CCl}_{3}$ spin adduct. Pure PBN-CCl ${ }_{3}$ was used as a standard to determine its retention time. The reconstructed mass chromatograms of $m / z$ 295, 297, and 299 from a pure $\mathrm{PBN}-\mathrm{CCl}_{3}$ sample were recorded (data not shown). The $m / z 295,297$, and 299 are the $[\mathrm{M}+\mathrm{H}]^{+\cdot}$ ions of the trichloromethyl PBN spin adduct.

The reconstructed mass chromatograms of $\mathrm{m} / \mathrm{z} 295$, 297, and 299 show that the retention time of $\mathrm{PBN}-\mathrm{CCl}_{3}$ is approximately $12.2 \mathrm{~min}$ when $\mathrm{CH}_{3} \mathrm{CN}: \mathrm{H}_{2} \mathrm{O}(65: 35)$ is used as a mobile phase at a flow rate of $0.2 \mathrm{~mL} / \mathrm{min}$ (spectrum not shown). The TS-MS spectrum that corresponds to this retention time shows isotope peaks at $m / z 295,297,299$, and 301. These are assigned to the protonated $\mathrm{PBN}-\mathrm{CCl}_{3}$ spin adduct (Figure 5). Peaks at

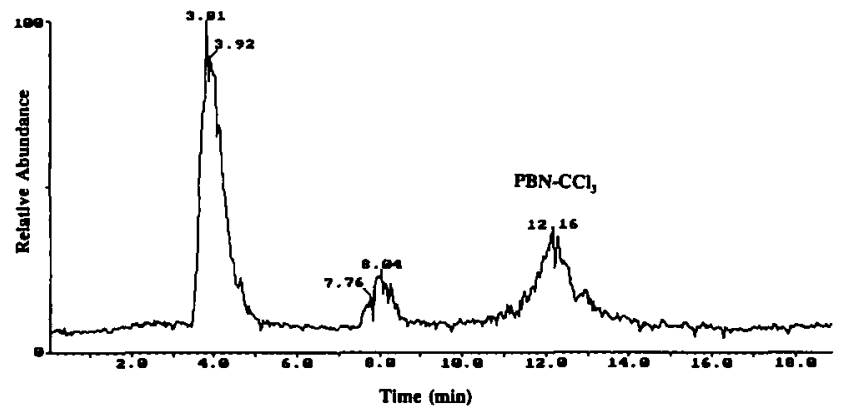

Figure 5. The thermospray mass spectrum at retention time about 12 min obtained during LC/TS-MS analysis of the pure $\mathrm{PBN}-\mathrm{CCl}_{3}$. 
$m / z 296,298,300$, and 302 are attributed to the hy-

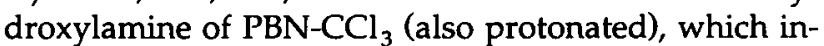
dicates that hydroxylamine was formed via thermospray ionization.

Figure 6 is a reconstructed mass chromatogram of $\mathrm{m} / \mathrm{z} 295$ from the hexane extract of rat liver microsomal dispersion incubated with $\mathrm{BrCCl}_{3}$ in the presence of 25-mM PBN. The mass spectrum of the component that eluted at $12.1 \mathrm{~min}$ shows $\mathrm{PBN}-\mathrm{CCl}_{3}$ isotope peaks at $m / z 295,297,299$, and 301 . The isotope peaks of the protonated hydroxylamine of $\mathrm{PBN}-\mathrm{CCl}_{3}$ also were observed at $m / z 296,298,300$, and 302 . The scan of protonated precursors of $\mathrm{m} / z 57$ recorded at the same retention time also exhibits two overlapping isotope patterns: $m / z$ 295, 297, 299, and 301 as well as 296, 298,300 , and 302. The product ion spectra of $\mathrm{m} / z 295$ and 297 showed fragments $m / z$ 178, 122, and 57, which suggests that the $[\mathrm{M}+\mathrm{H}]^{+\cdot}$ ion loses $\mathrm{HCCl}_{3}$ to produce protonated $\mathrm{PBN}(m / z$ 178), which further decomposes to $m / z 122$ (Scheme II).

Repetition of the experiment with $\mathrm{PBN}-d_{9}$ gave very similar chromatographic and mass spectrometric results. The reconstructed mass chromatogram of $\mathrm{m} / \mathrm{z}$ 304 shows that the retention time of PBN- $d_{9}-\mathrm{CCl}_{3}$ is about $12 \mathrm{~min}$. The mass spectrum of this peak is shown in Figure 7. The peaks at $m / z$ 304, 306, 308, and 310 are attributed to protonated PBN- $d_{9}-\mathrm{CCl}_{3}$, and the peaks at $m / z 305,307,309$, and 311 are due to the protonated hydroxylamine of $\mathrm{PBN}-d_{9}-\mathrm{CCl}_{3}$.

In the spectrum of precursors of $m / z 66$, peaks at $m / z$ 304, 306, 308, and 310 and at $m / z 305,307,309$, and 311 were observed. Again, $m / z$ 289, 291, and 293 are attributed to $\mathrm{PBN}-d_{9}-\mathrm{CCl}_{3}$, which loses an oxygen and abstracts a proton. The product ion spectra of both $m / z 304$ and 306 showed peaks at $m / z 187,123$, and 66 (spectrum not shown). Thus $m / z 187$ can be assigned to the protonated $\mathrm{PBN}-d_{9}-\mathrm{CCl}_{3}$ after losing $\mathrm{HCCl}_{3}$ and $m / z 123$ is protonated $\mathrm{PBN}-d_{9}-\mathrm{CCl}_{3}$ after losing both $\mathrm{HCCl}_{3}$ and the iso-butylene group. Therefore, the major fragmentation of the $\mathrm{PBN}-\mathrm{CCl}_{3}$ spin adduct involves formation of tert-butyl ions or loss of a $\mathrm{HCCl}_{3}$ neutral molecule that leaves protonated PBN as the positive charged fragment, with further loss of isobutylene by a McLafferty-like rearrangement.

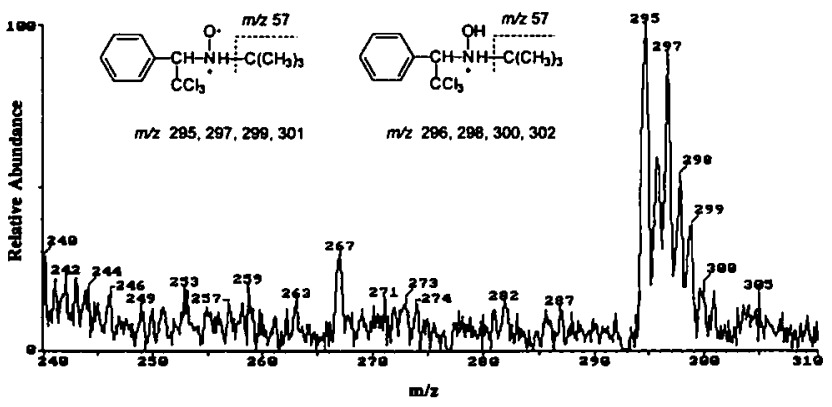

Figure 6. The reconstructed mass chromatogram of $\mathrm{m} / \mathrm{z} 295$ obtained during LC/TS-MS of pure $\mathrm{PBN}-\mathrm{CCl}_{3}$ of a hexane extract from rat liver microsomes incubated with $\mathrm{BrCCl}_{3}$ in the presence of 25-mM PBN.

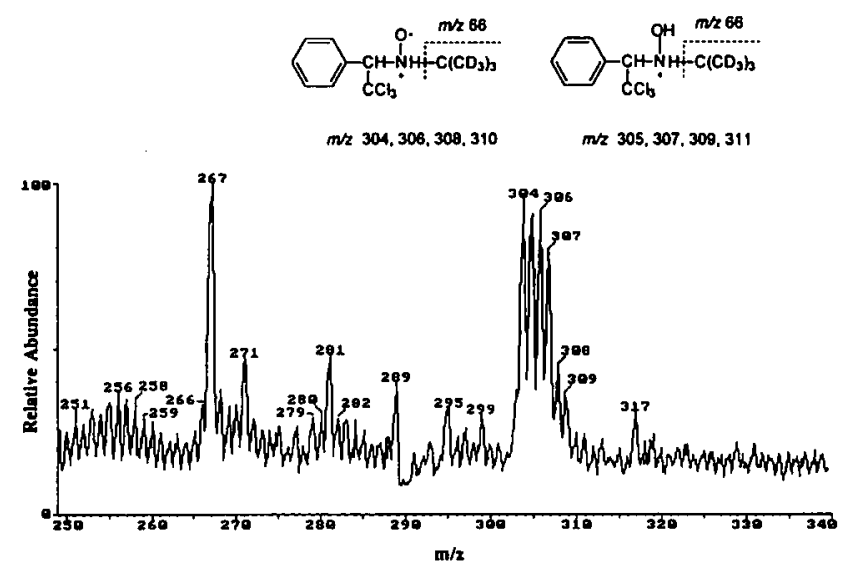

Figure 7. The thermospray mass spectrum at retention time about $12 \mathrm{~min}$ obtained during LC/TS-MS analysis of a hexane extract from rat liver microsomes incubated with $\mathrm{BrCCl}_{3}$ in the presence of $10-\mathrm{mM}$ PBN- $d_{9}$.

\section{Conclusion}

A long-standing problem in the mass spectrometry identification and structural analysis of spin adducts has been separation. Without prior liquid and/or gas chromatrography separation, one cannot usually obtain any information about spin adduct structure from conventional mass spectrometry. In this study EI with tandem mass spectrometry, combined with the use of deuterated PBN, has been shown to be a useful technique for the identification of $\mathrm{PBN}-\mathrm{CCl}_{3}$ spin adducts and other PBN spin adducts. In the identification of PBN spin adducts, the scan of precursors of $\mathrm{m} / \mathrm{z} 57$ was useful as a screening step. The use of deuterated PBN with a scan of precursors of $m / z 66$ can provide more definitive information with regard to PBN spin adducts - both known and unknown spin adducts. If the product ion spectra of these spin adducts provide sufficient structural information, it is possible to assign all spin adducts. However, sometimes the product ion

Fragmentation of $\mathrm{PBN}-\mathrm{CCl}_{3}$

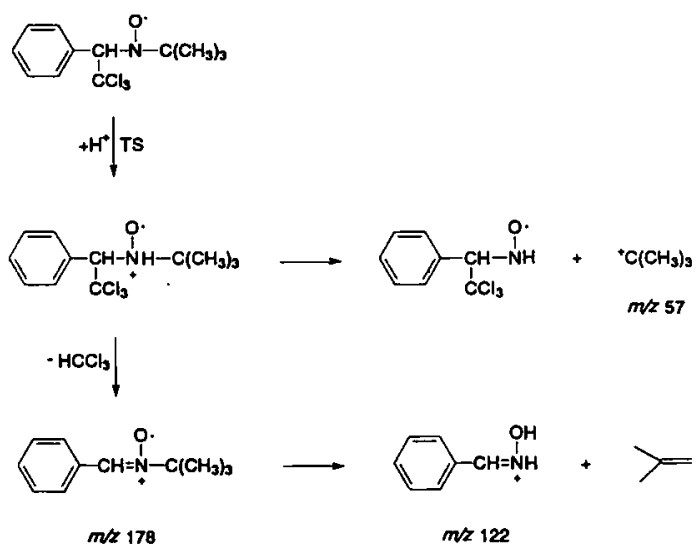

Scheme II. Fragmentation of $\mathrm{PBN}-\mathrm{CCl}_{3}$. 
spectrum of the PBN spin adduct under EI conditions does not give sufficient fragments for deduction of the structure; for example, the $\mathrm{m} / \mathrm{z} 57$ fragment is the only product ion from $\mathrm{PBN}-\mathrm{CCl}_{3}$.

Thermospray tandem mass spectrometry is a soft ionization method that also appears useful for detecting unstable spin adducts. The product ion spectrum moreover can give more fragments than EI tandem mass spectrometry. This is presumably associated with the fact that we are dealing with a different precursor ion $\left(\mathrm{MH}^{+}\right.$, rather than $\mathrm{M}^{+\cdot}$ in EI experiments). For example, the product ion spectrum of $\mathrm{PBN}-\mathrm{CCl}_{3}$ gave fragments that correspond to loss of $\mathrm{CHCl}_{3}$ and futher loss of isobutylene molecules.

In summary, we used tandem mass spectrometry methodology to show that mass spectrometry can be used for the detection and identification of spin adducts of PBN. In contrast to the previous studies [31], the concentration of spin adducts obtained from the biological system was high enough to be detected by mass spectometry.

\section{Acknowledgments}

This work was supported by funds provided by an NIH grant (RR05517) from the Center for Research Resources. We also gratefully acknowledge the ongoing support from the Oklahoma Medical Research Foundation, which was responsible for the purchase of the tandem mass spectrometer used in this work. Dr. Masana Arimura was on sabbatical leave and derived partial support from the Institute of Chemistry, College of General Education, Osaka University, Osaka, Japan.

\section{References}

1. Janzen, E. G. Acc. Chem. Res. 1971, 4, 31-40.

2. Perkins, M. J. Adv. Phys. Org. Chem. 1980, 17, 1-64.

3. Janzen, E. G. Free Radicals in Biol. 1980, 4, 115-154.

4. Finklestein, E.; Rosen, G. M.; Rauckman, E. J. Arch. Biochem. Biophys. 1980, 200, 1-16.

5. Kalyanaraman, B. Rev. Biochem. Toxicol. 1982, 4, 73-139.

6. Mottley, C.; Mason, R. P. Biol. Magn. Reson. 1989, 8, 489-546.

7. Iwahashi, H.; Albro, P. W.; McGown, S. R.; Tomer, K. B.; Mason, R. P. Arch. Biochem. Biophys. 1991, 285, 172-180.

8. Janzen, E. G. Methods Enzymol. 1984, 105, 188-198.

9. Janzen, E. G.; Krygsman, P. H.; Haire, D. L. Biomed. Environ. Mass Spectrom. 1988, 15, 111-116.

10. Noda, A.; Noda, H.; Ohno, K.; Sendo, T.; Misaka, A.; Kanazawa, Y.; Isobe, R.-I.; Hirata, M. Biochem. Biophys. Res. Commun. 1985, 133, 1086-1091.

11. Janzen, E. G.; Weber, J. R.; Haire, D. L.; Fung, D. M. Anal. Lett. 1985, 18, 1749-1757.

12. Mikami, N.; Takahashi, N.; Yamada, H.; Miyamoto, J. Pestic. Sci. 1985, 16, 101-112.

13. Abe, K.; Suezawa, H.; Hirota, M. J. Chem. Soc., Perkin Trans. 2 1984, 29-34.

14. Ortiz de Montellano, P. R.; Augusto, O.; Viola, F.; Kunze, K. L. J. Biol. Chem. 1983, 258, 8623-8629.

15. Watanabe, T.; Yoshida, M.; Fujiwara, S.; Abe, K.; Onoe, A.; Hirota, M.; Igarashi, S. Anal. Chem. 1982, 54, 2470-2474.

16. Sinha, B. K.; Motten, A. G. Biochem. Biophys. Res. Commun. 1982, 105, 1044-1051.

17. Suezawa, H.; Abe, K.; Hirota, M.; Ishii, T. Chem. Lett. 1981, 1049-1052.
18. Hill, H. A. O.; Thornalley, P. J. FEBS Lett. 1981, 125, 235-238.

19. Janzen, E. G.; Towner, R. A.; Krygsman, P. H.; Haire, D. L. Free Radical Res. Commun. 1990, 9, 343-351.

20. Janzen, E. G.; Towner, R. A.; Krygsman, P. H. Free Radical Res. Commun. 1990, 9, 353-360.

21. Krygsman, P. H.; Janzen, E. G.; Towner, R. A.; Haire, D. L. Anal. Lett. 1989, 22, 1009-1020.

22. Iwahashi, H.; Parker, C. E.; Mason, R. P.; Tomer, K. B. Rapid Commun. Mass. Spectrom. 1990, 4, 352-354.

23. Iwahashi, H.; Parker, C. E.; Mason, R. P.; Tomer, K. B. Biochem. J. 1991, 276, 447-453.

24. Parker, C. E.; Iwahashi, H.; Tomer, K. B. I. Am. Soc. Mass Spectrom. 1991, 2, 413-418.

25. Iwahashi, H.; Parker, C. E.; Mason, R. P.; Tomer, K. B. Anal. Chem. 1992, 64, 2244-2252.

26. Iwahashi, H.; Albro, P. W.; McGown, S. R.; Tomer, K. B.; Mason, R. P. Arch. Biochem. Biophys. 1991, 285, 172-180.

27. Makino, K.; Hatano, H. Chem. Lett. 1979, 119-122.

28. Makino, K.; Moriyama, F.; Hatano, H. J. Chromatogr. 1985, $332,71-106$.

29. Iwahashi, H.; Ikeda, A.; Negoro, Y.; Kido, R. Biochem. J. 1986, 236, 509-514.

30. Sugata, R.; Iwahashi, H.; Ishii, T.; Ryo, K. J. Chromatogr. 1989, $487,9-16$.

31. Iwahashi, H.; Parker, C. E.; Tomer, K. B.; Mason, R. P. Free Radical Res. Commun. 1992, 16, 295-301.

32. Janzen, E. G.; Lindsay, D. A.; Haire, D. L. I. Am. Chem. Soc. 1990, 112, 8279-8284.

33. Lai, E. K.; McCay, P. B.; Noguchi, T.; Fong, K. L. Biochem. Pharmacol. 1979, 28, 2231-2235.

34. Poyer, J. L.; McCay, P. B.; Lai, E. K.; Janzen, E. G.; Davis, E. R. Biochem. Biophys. Res. Commun. 1980, 94, 1154-1160.

35. McCay, P. B.; Lai, E. K.; Poyer, J. L.; DuBose, C. M.; Janzen, E. G. J. Biol. Chem. 1984, 259, 2135-2143.

36. Albano, E.; Lott, K. A. K.; Slater, T. F.; Stier, A.; Symons, M. C. R.; Tomasi, A. Biochem. J. 1982, 204, 593-603.

37. Tomasi, A.; Albano, E.; Bini, A.; Botti, B.; Slater, T. F.; Vannini, V. Toxicol. Pathol. 1984, 12, 240-246.

38. Poyer, J. L.; Floyd, R. A.; McCay, P. B.; Janzen, E. G.; Davis, E. R. Biochim. Biophys. Acta 1978, 539, 402-409.

39. Kalyanaraman, B.; Mason, R. P.; Perez-Reyes, E.; Chignell, C. F.; Wolf, C. R.; Philpot, R. M. Biochem. Biophys. Res. Commun. 1979, 89, 1065-1072.

40. Janzen, E. G.; Stronks, H. J.; DuBose, C. M.; Poyer, J. L.; McCay, P. B. Environ. Health Perspect. 1985, 64, 151-170.

41. Janzen, E. G.; Towner, R. A.; Haire, D. L. Free Radical Res. Commun. 1987, 3, 357-364.

42. Janzen, E. G.; Towner, R. A.; Brauer, M. Free Radical Res. Commun. 1988, 4, 359-369.

43. Bush, K. L.; Glish, G. L.; McLuckey, S. A. Mass Spectrometry/Mass Spectrometry: Techniques and Applications of Tandem Mass Spectrometry; VCH Publishers: New York, 1988.

44. MaLafferty, F. W. Interpretation of Mass Spectra, 3rd ed.; University Science Books: Mill Valley, CA, 1980; p 16.

45. Janzen, E. G.; Chen, G.; Bray, T. M.; Reinke, L. A.; Poyer, J. L.; McCay, P. B. J. Chem. Soc., Perkin Trans. 2, 1993, 1983-1989.

46. Kotake, Y.; Janzen, E. G. J. Am. Chem. Soc. 1991, 113, 9503-9506.

47. Janzen, E. G.; Kotake, Y; Hinton, R. D. Free Radical Biol. Med. 1992, 12, 169.

48. Reinke, L. A.; Mailey, S. M.; Rau, J. M.; McCay, P. B. Free Radical Res. 1994, 20, 51-60.

49. Reinke, L. A.; Rau, J. M.; McCay, P. B. Free Radical Biol. Med. 1994, 16, 485-492.

50. Janzen, E. G.; DuBose, C. M. Anal. Lett. 1993, 26, 2661-2666. 